\title{
Asymmetric Temperature Profiles in Joule-Heated in Aluminum Nanowires
}

\author{
B. C. Regan ${ }^{1}$, Brian Zutter ${ }^{1}$, William A. Hubbard ${ }^{1}$, Shaul Aloni ${ }^{2}$, and Matthew Mecklenburg ${ }^{3}$ \\ 1. Department of Physics and Astronomy \& California NanoSystems Institute, University of California, \\ Los Angeles, CA, USA \\ 2. Molecular Foundry, Lawrence Berkeley National Laboratory, Berkeley, CA, USA \\ 3. Center for Electron Microscopy and Microanalysis, University of Southern California, Los Angeles, \\ CA, USA
}

Subject to direct current (DC) Joule heating, a straight, suspended wire that is heat-sunk at its ends will produce a symmetric temperature distribution that peaks in the middle of the wire. This temperature distribution is independent of the sign of the DC current through the wire. We have observed asymmetric temperature profiles in Joule heated aluminum nanowires (60 nm thick by $30 \mathrm{~nm}$ wide): the location of the maximum temperature switches sides around the midpoint of the wire depending on the sign of the current through the wire. Simulations of the wire's temperature profile indicate that the asymmetry could arise from an unexpectedly large value of the nanowire's thermoelectric Thomson coefficient.

We measured the temperature in microfabricated aluminum nanowires using: a transmission electron microscope (JEOL JEM-2100F) operated at a high tension of $80 \mathrm{kV}$, an electron energy loss spectrometer (Gatan Quantum SE GIF) with an energy dispersion of $25 \mathrm{meV} / \mathrm{pixel}$, and an electrical biasing sample holder (Hummingbird Scientific). Valence electron energy loss spectroscopy (EELS) spectrum images were acquired while supplying constant power to an aluminum nanowire. The temperature distribution in the silicon-nitride-supported aluminum nanowires was determined from the plasmon energy shifts through plasmon energy expansion thermometry (PEET) [1, 2]. Maps of the temperature of an aluminum nanowire show its temperature increasing monotonically as the current magnitude varied from $120 \mu \mathrm{A}$ to $310 \mu \mathrm{A}$ (Figure 1). (The power supplied to the complete circuit, including the leads, varied from $30 \mu \mathrm{W}$ and $210 \mu \mathrm{W}$ in $30 \mu \mathrm{W}$ intervals.) The maximum temperature in the aluminum wire reached $\sim 100 \mathrm{~K}$ above ambient at the maximum power of $210 \mu \mathrm{W}$.

The acquisitions with a negatively (positively) signed current shows a maximum temperature near the top (bottom) connection in Figure 1, an asymmetry that is not seen in larger Joule-heated aluminum nanowires [1]. With an applied current of $290 \mu \mathrm{A}$ the hottest part of the wire, $80 \mathrm{~K}$ above room temperature, appeared $\sim 1 \mu \mathrm{m}$ from the center of the $\sim 4 \mu \mathrm{m}$ long wire. We generated simulated temperature profiles (Figure 2) assuming the aluminum nanowire has a thermal conductivity of $95 \mathrm{~W} / \mathrm{m}$ $\mathrm{K}$, a Thomson coefficient of $-26 \mu \mathrm{V} / \mathrm{K}$, and an electrical resistivity calculated using the WiedemannFranz law. The assumed thermal conductivity lies within a factor of three of the measured bulk value of $240 \mathrm{~W} / \mathrm{m}-\mathrm{K}$, but the Thomson coefficient is much larger than the bulk value of $-26 \mathrm{nV} / \mathrm{K}$ [3]. The simulations account for the silicon nitride membrane support and show a good agreement with our data. More work is needed to understand the origin of these asymmetric temperature profiles, but these results may give an example of how the thermal transport properties of materials change at the nanoscale.

References:

[1] M Mecklenburg et al, Science 347 (2015), p. 629-632.

[2] M P Seah and G C Smith, Journal of Materials Science 21 (1986), p. 1305-1309. 
[3] D De et al, Journal of Applied Physics, 54 (1983), p. 4022-4027.

[4] This work was supported by FAME, one of six centers of STARnet, a Semiconductor Research Corporation program sponsored by MARCO and DARPA. Data presented were acquired at the Center for Electron Microscopy and Microanalysis at the University of Southern California.
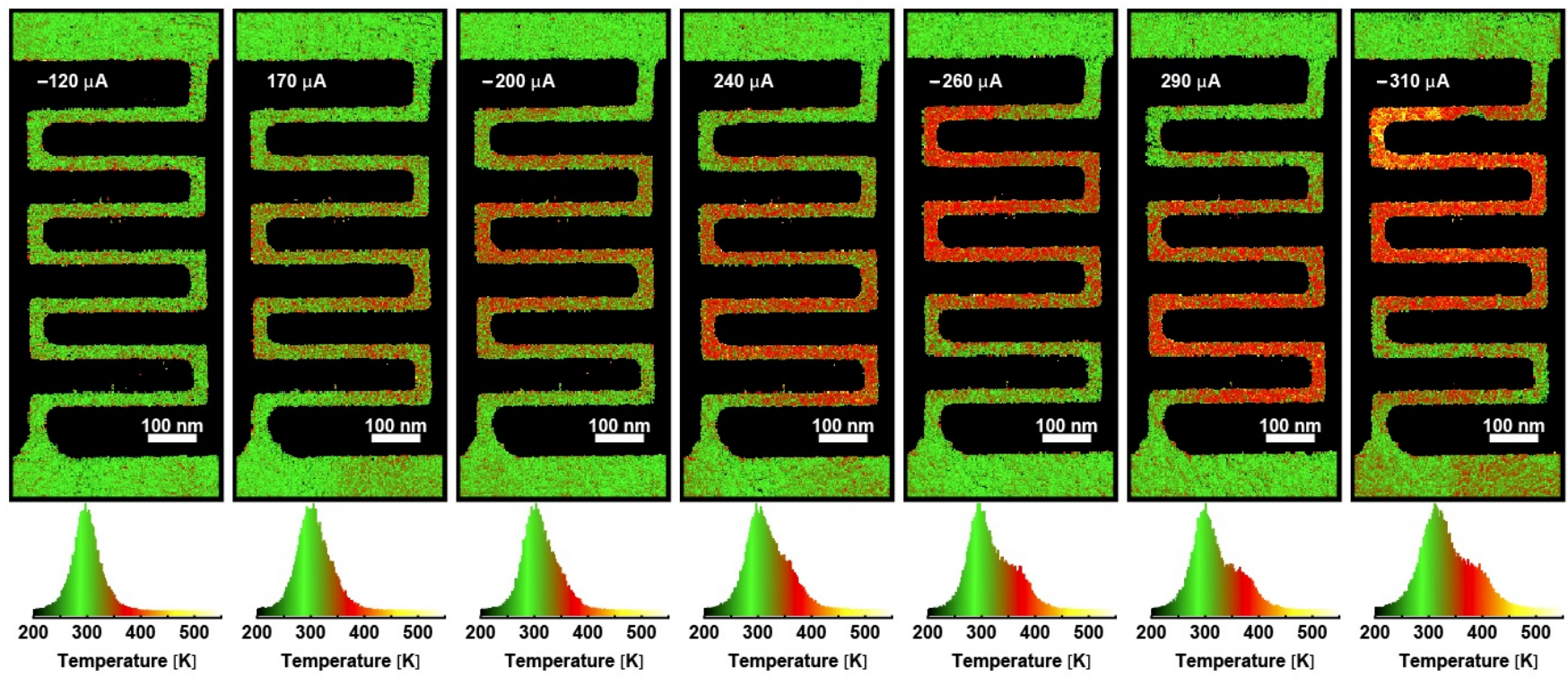

Figure 1. Temperature maps, each 359 pixels tall by 159 pixels wide in size, with $3 \mathrm{~nm}$ sized pixels, of Joule-heated aluminum nanowires. The magnitude and sign of the electrical current passing through the nanowire is indicated on each map. Below each map is a histogram of the determined temperatures. The colors of the histograms indicate the single temperature scale used in the maps.
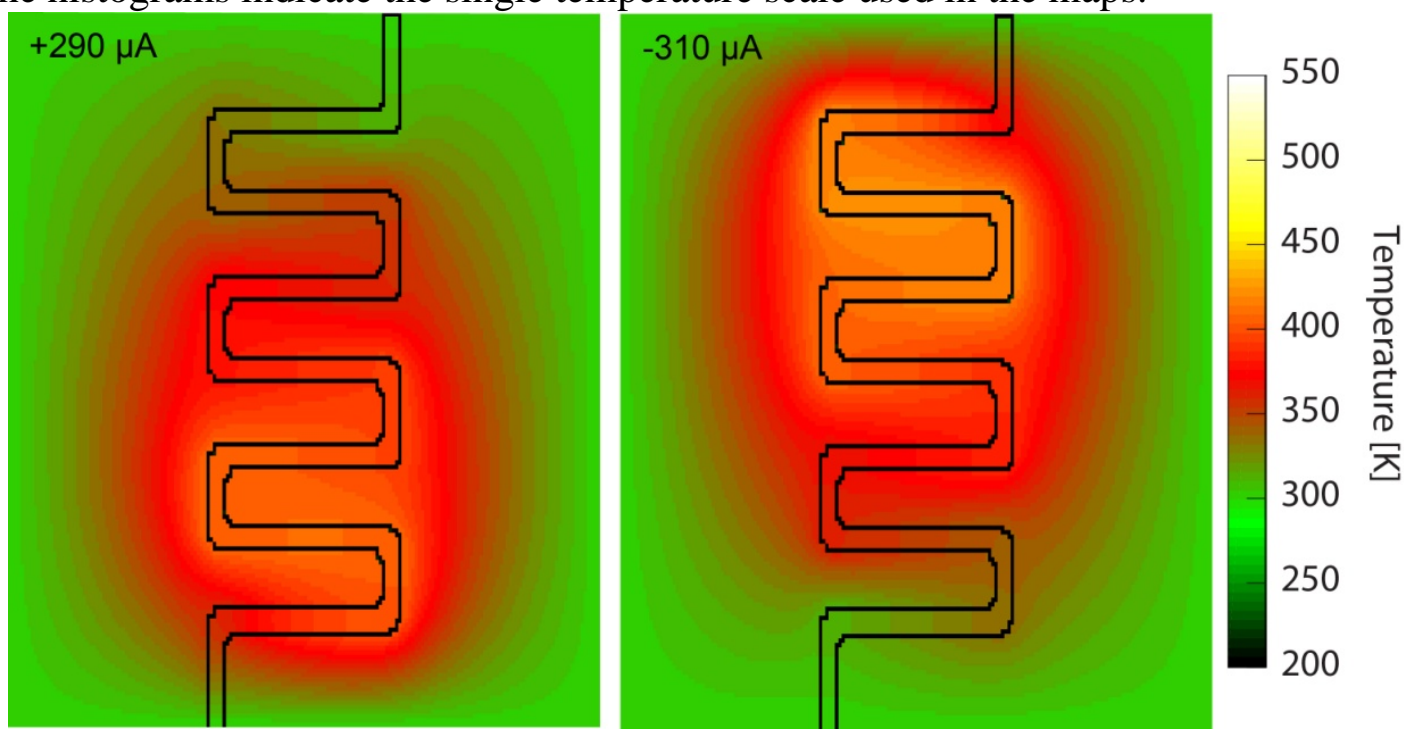

Figure 2. Simulations of the temperature in and around an aluminum nanowire. The outline of the aluminum nanowire is shown in black. The temperature maps are 182 pixels tall and 158 pixels wide with an $8 \mathrm{~nm}$ pixel size. One simulation is for $+290 \mu \mathrm{A}$ passing through the nanowire, and the other is for $-310 \mu \mathrm{A}$. According to our sign convention, a positive current flows through the nanowire when the top contact is biased to positive voltage relative to the bottom contact. 